\title{
NEUMANN EIGENVALUE SUMS ON TRIANGLES ARE (MOSTLY) MINIMAL FOR EQUILATERALS
}

\author{
R. S. LAugesen, Z. C. PAN And S. S. Son
}

Abstract. We prove that among all triangles of given diameter, the equilateral triangle minimizes the sum of the first $n$ eigenvalues of the Neumann Laplacian, when $n \geqslant 3$.

The result fails for $n=2$, because the second eigenvalue is known to be minimal for the degenerate acute isosceles triangle (rather than for the equilateral) while the first eigenvalue is 0 for every triangle. We show the third eigenvalue is minimal for the equilateral triangle.

Mathematics subject classification (2010): Primary 35P15. Secondary 35J20.

Keywords and phrases: Isodiametric, isoperimetric, free membrane.

\section{REFERENCES}

[1] B. Andrews And J. Clutterbuck, Proof of the fundamental gap conjecture. Preprint, 2010. arXiv: 1006.1686

[2] P. Antunes And P. Freitas, A numerical study of the spectral gap, J. Phys. A 41 (2008), 055201, $19 \mathrm{pp}$.

[3] M. S. Ashbaugh, Isoperimetric and universal inequalities for eigenvalues. In: Spectral theory and geometry (Edinburgh, 1998), 95-139, London Math. Soc. Lecture Note Ser., 273, Cambridge Univ. Press, Cambridge, 1999.

[4] C. BAndle, Isoperimetric Inequalities and Applications. Pitman, Boston, Mass., 1979.

[5] R. BAÑuelos AND K. BuRdZY, On the "hot spots" conjecture of J. Rauch, J. Funct. Anal., 164 (1999), 1-33.

[6] A. Henrot, Extremum Problems for Eigenvalues of Elliptic Operators. Frontiers in Mathematics. Birkhäuser Verlag, Basel, 2006.

[7] B. KAWOHL, Rearrangements and convexity of level sets in PDE. Lecture Notes in Mathematics, 1150. Springer-Verlag, Berlin, 1985.

[8] S. Kesavan, Symmetrization \& Applications. Series in Analysis, 3. World Scientific Publishing, Hackensack, NJ, 2006.

[9] P. KRÖGER, Estimates for sums of eigenvalues of the Laplacian, J. Funct. Anal., 126 (1994), 217-227.

[10] M. G. LamÉ, Leçons sur la Théorie Mathématique de L'Élasticité des Corps Solides. Deuxième édition. Gauthier-Villars, Paris, 1866.

[11] R. S. Laugesen And B. A. Siudeja, Minimizing Neumann fundamental tones of triangles: an optimal Poincaré iequality, J. Differential Equations, 249 (2010), 118-135.

[12] R. S. LAUGESEN AND B. A. SiUdeJA, Sums of Laplace eigenvalues - rotationally symmetric maximizers in the plane. J. Funct. Anal., 260 (2011), 1795-1823.

[13] R. S. LAUGeSEn AND B. A. SiUdejA, Dirichlet eigenvalue sums on triangles are minimal for equilaterals. Preprint. arXiv:1008.1316.

[14] J. Mathews And R. L. Walker, Mathematical Methods of Physics. Second edition. W. A. Benjamin, New York, 1970.

[15] B. J. MCCARTin, Eigenstructure of the equilateral triangle. II. The Neumann problem. Math. Probl. Eng., 8 (2002), 517-539.

[16] L. E. Payne And H. F. Weinberger, An optimal Poincaré inequality for convex domains, Arch. Rational Mech. Anal., 5 (1960), 286-292. 
[17] M. A. PINSKY, Completeness of the eigenfunctions of the equilateral triangle, SIAM J. Math. Anal., 16 (1985), 848-851.

[18] G. Pólya AND G. SZEgô, Isoperimetric Inequalities in Mathematical Physics. Princeton University Press, Princeton, New Jersey, 1951. 